\title{
Exercise-induced knee joint laxity in distance runners
}

\author{
H.V. Johannsen, MD, T. Lind, MD, B.W. Jakobsen, MD and K. Krøner, MD
}

Accident Analysis Centre, Division of Sports Traumatology, Department of Orthopaedic Surgery, Aarhus County Hospital, University of Aarhus, Denmark

\begin{abstract}
The objectives of this study were to evaluate the effect of exercise on knee joint laxity. If exercise induced laxity is physiological, incorporation of this quality into a ligament replacement material would be indicated. Twenty recreational long distance runners average age 41 (range 24 to 50 yr) were tested before and immediately after 30 minutes of running. Using a computerized goniometer type instrument (Acufex KSS), knee flexion, axial tibial rotation and anterior-posterior tibial displacement were simultaneously recorded, while the runners underwent tests of static as well as dynamic knee joint laxity.

At 30 degrees of knee flexion, a maximum increase of $\mathbf{1 6}$ per cent in mean total anterior-posterior laxity post-exercise was found. At the examination 30 minutes post-exercise, laxity at 30 degrees of knee flexion was still increased. However, laxity at 90 degrees of knee flexion had decreased to pre-exercise levels or below. Anterior tibial displacement, recorded during eccentric quadriceps activity $(0$ to 90 degrees of knee flexion) with weights attached to the foot, showed a maximum of 18 per cent increase in total anteriorposterior laxity post-exercise. It is suggested that the laxity increase is caused in part by a true ligamentous laxity increase, and in part by a decreased resting tone of the fatigued muscles.
\end{abstract}

Keywords: Anterior-posterior knee joint laxity, torsional knee laxity, exercise

\section{Introduction}

Knee joint stability under physiological conditions is maintained by the complex interactions of several factors: ligament and other soft tissue restraints, active muscular control and joint surface-contact forces. Transient increased laxity or compliance following cyclic deformation of connective tissues has been documented in laboratory tests ${ }^{1,2}$. In vivo, muscle substance as well as ligamentous restraints would be expected to demonstrate visco-elastic behaviour as they are composed of collagen and other structural proteins. Therefore, a time-dependent and stress-dependent elongation response of these tissues, when subjected to repetitive loading, could be expected.

Recent studies have documented increased torsional, valgus and anterior-posterior laxity in the knee following exercise ${ }^{3-6}$. In all of these studies the laxity increases found were increases in passive or static laxity at fixed joint positions.

An understanding of the nature and degree of laxity changes may provide insight into a possible association between exercise-induced laxity and subsequent ligamentous injury. A further clinical implication is that, if exercise-induced laxity is physiological, incorporation of this quality into a ligament replacement material would be indicated.

The objectives of this study were to evaluate the effect of exercise on knee joint laxity, using both tests of static laxity for determination of anterior-posterior and torsional laxities, as well as dynamic laxity tests, for recording of rotational and anterior-posterior tibial displacement resulting from hamstring or quadriceps activity. Furthermore, an assessment of the time chronology of recovery through repeated measurements was attempted.

\section{Testing apparatus}

All laxity measurements were made using a commercially available laxity testing device: the Acufex Knee Signature System (Acufex Microsurgical Inc., Norwood, USA). Lightweight frames are attached to the tibia and femur by means of rubber straps. A linking section, allowing free knee joint motion, connects the tibial and femoral frames. Two small potentiometers are integrated into this section: one measuring degrees of knee flexion, the other axial tibial rotation relative to the femur.

At the proximal end of the tibial frame a small spring-loaded plate, in contact with the patella and connected to an additional potentiometer, measures anterior-posterior tibial displacement by measuring the distance between the tuberositas tibiae and patella, and hence femoral condyles. By means of a handle with an incorporated force, sensitive straingauge force can be applied to the tibia and force versus displacement curves obtained.

All data are relayed through cables to a mini-computer and displayed as curves on the screen simultaneously with the performance of the test. A plotter is supplied to give paper copies of test results if needed.

The tibial and femoral frames can be securely fixed, allowing a variety of tests to be performed. Subjects can be tested sitting relaxed, legs fixed at various angles of knee flexion, or while performing isometric 
or eccentric quadriceps or hamstring exercises. The apparatus even allows recording during walking, jumping or the like, within range of the cables. During the tests of rotational laxity, axial tibial rotational force was applied manually, using a spring-loaded torque wrench firmly secured to the subject's foot with a boot.

\section{Test protocol}

Seven different tests were performed:

1 Passive anterior and posterior tibial displacement at 30 degrees of knee flexion (Force 0-200 N)

2 Anterior tibial displacement and axial rotation caused by maximal voluntary isometric quadriceps contraction against resistance at 30 degrees of knee flexion

3 Passive anterior and posterior tibial displacement at 90 degrees of knee flexion (Force $0-200 \mathrm{~N}$ )

4 Posterior tibial displacement and axial rotation caused by maximal voluntary isometric hamstring contraction against resistance at 90 degrees of knee flexion

5 Passive internal and external tibial rotation at 90 degrees of knee flexion and 5 Newton meters of applied torque

6 Anterior tibial displacement during eccentric quadriceps activity ( 0 to 90 degrees of knee flexion) with 10 per cent of body weight attached to the foot

7 Tibial displacement and rotation during walking (one complete step).

During exercises 1-6 the subjects were seated on a quadriceps extension table. All measurements were made both prior and immediately after the running period. In addition, tests 1 and 3 were repeated 30 minutes after completion of exercises. Since only one knee at a time could be tested, one knee only per runner was tested to ensure that the testing could be performed immediately post-exercise. The left or right knee was chosen at random. The paired, one-tailed $t$ test was used in comparing pre- with post-exercise laxity.

\section{Test group}

Twenty recreational long distance runners (eleven men and nine women) volunteered for the test. This test group was chosen because they were capable runners and generally in excellent physical condition, as demonstrated by the fact that 17 of these runners had completed one or several marathons within the last year. The group averaged 41 years of age (range 24-50). None of the subjects had had any previous knee injury, and at physical examination no knee abnormalities were found. The subjects were asked to run at 'sub-maximum speed' for 30 minutes. Warming-up or cooling-down exercises were not allowed. The track was flat and the temperature between 5 and $10^{\circ} \mathrm{C}$.

\section{Results}

A summary of the static anterior-posterior, joint laxity data at 30 and 90 degrees of knee flexion are given in Table 1 and Table 2 respectively. With few exceptions, all mean values of passive laxity showed increases
Table 1. Pre- and post-exercise values of applied force versus anterior and posterior tibial displacement at 30 degrees of knee flexion in 20 athletes ( 20 knees). Mean values and one standard deviation

\begin{tabular}{lcccc}
\hline & $50 \mathrm{~N}$ & $100 \mathrm{~N}$ & $150 \mathrm{~N}$ & $200 \mathrm{~N}$ \\
\hline & Ant./Post. & Ant./Post. & Ant./Post. & Ant./Post. \\
Pre-exercise & $4.0 / 2.2$ & $7.1 / 3.8$ & $8.7 / 4.9$ & $9.7 / 5.8$ \\
& $( \pm 2.0)$ & $( \pm 2.8)$ & $( \pm 5.4)$ & $( \pm 3.7)$ \\
Post-exercise & $4.8 / 2.4^{*}$ & $8.1 / 4.5^{* *}$ & $10.1 / 5.8^{* * *}$ & $11.1 / 6.7^{* *}$ \\
& $( \pm 1.9)$ & $( \pm 2.9)$ & $( \pm 3.9)$ & $( \pm 4.1)$ \\
30minutes & $4.6 / 2.0$ & $7.8 / 3.6$ & $9.4 / 4.9$ & $10.4 / 5.8$ \\
post-exercise & $( \pm 2.0)$ & $( \pm 2.8)$ & $( \pm 3.7)$ & $( \pm 4.1)$ \\
\hline
\end{tabular}

Ant. anterior (millimetres); Post. posterior (millimetres)

In parentheses: one standard deviation (total ant.-post. displacement) Pre- versus post-exercise total anterior-posterior displacement: ${ }^{* * *} P<0.005 ;{ }^{* *} P<0.01{ }^{*} P \pm 0.025$

Table 2. Pre- and post-exercise values of applied force versus anterior and posterior tibial displacement at 90 degrees of knee flexion in 20 athletes ( 20 knees). Mean values and one standard deviation

\begin{tabular}{lcccc}
\hline & $50 \mathrm{~N}$ & $100 \mathrm{~N}$ & $150 \mathrm{~N}$ & $200 \mathrm{~N}$ \\
\hline & Ant./Post. & Ant./Post. & Ant./Post. & Ant./Post. \\
Pre-exercise & $2.8 / 1.4$ & $5.1 / 2.6$ & $7.0 / 3.8$ & $7.8 / 4.8$ \\
& $( \pm 1.4)$ & $( \pm 2.5)$ & $( \pm 3.4)$ & $( \pm 3.5)$ \\
Post-exercise & $3.0 / 1.7^{*}$ & $5.4 / 3.3^{*}$ & $6.8 / 4.6$ & $7.6 / 5.7$ \\
& $( \pm 1.7)$ & $( \pm 2.9)$ & $( \pm 3.7)$ & $( \pm 4.2)$ \\
30 minutes & $2.6 / 1.5$ & $4.5 / 2.8$ & $5.8 / 3.8$ & $6.7 / 4.7$ \\
post-exercise & $( \pm 1.5)$ & $( \pm 2.5)$ & $( \pm 3.3)$ & $( \pm 4.0)$ \\
\hline
\end{tabular}

Ant. anterior (millimetres); Post. posterior (millimetres)

In parentheses: one standard deviation (total ant.-post. displacement)

Pre- versus post-exercise total anterior-posterior displacement:

${ }^{*} P<0.025$

post-exercise, even if changes were not always statistically significant. A graphic presentation of the central data from these tables is given in Figure 1 and Figure 2, illustrating the different patterns of laxity increases following exercise and laxity decreases following the recovery period.

Quadriceps active, anterior tibial displacement at 30 degrees of flexion (test 2) did not demonstrate lengthening after exercise; a pre-exercise mean displacement of $7.9 \pm 2.0 \mathrm{~mm}$ was found, post-exercise $7.8 \pm 2.0 \mathrm{~mm}$. Tibial axial rotation during this exercise increased slightly, but the difference was not statistically significant $(5.5 \pm 2.6$ mean pre-exercise degrees of tibial rotation versus $6.0 \pm 3.0$ degrees post-exercise). Hamstring active, posterior tibial displacement at 90 degrees of knee flexion (test 4 ) decreased by 6.3 per cent from pre- to post-exercise (from $3.8 \pm 1.1 \mathrm{~mm}$ to $3.6 \pm 1.2 \mathrm{~mm}$ ); tibial axial rotation also decreased slightly during this manoeuvre (from $3.6 \pm 1.8$ to $3.3 \pm 1.7$ degrees of rotation or 7.0 per cent). These differences are not statistically significant.

Passive axial tibial rotation at 90 degrees of knee flexion increased only insignificantly from 17.9 44.9 degrees to $18.8 \pm 6.3$ degrees post-exercise $(5.0$ per cent).

Anterior-posterior tibial displacement measured during walking showed only a moderate post-exercise 


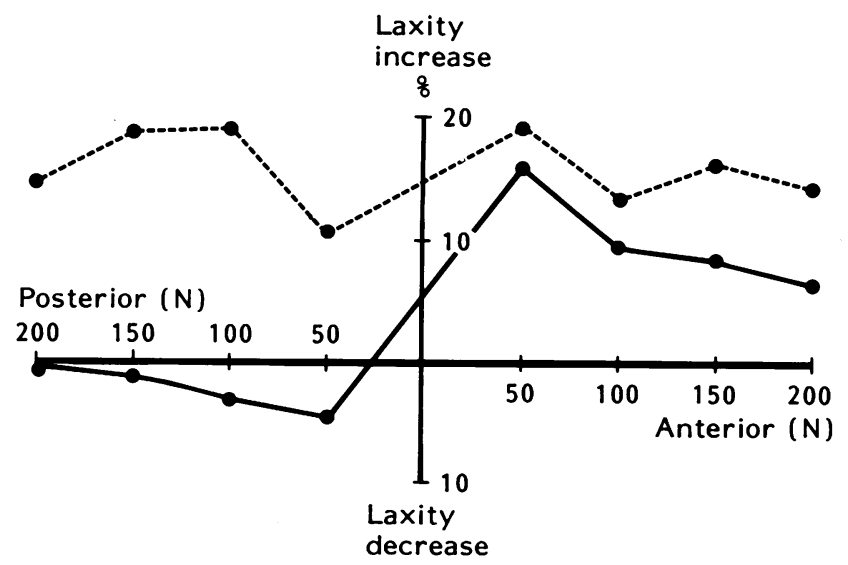

Figure 1. Force (Newton) versus anterior and posterior tibial displacement at 30 degrees of knee flexion: percentage increase or decrease of mean values relative to pre-exercise mean values (20 knees)

- - Immediately post-exercise

-30 minutes post-exercise

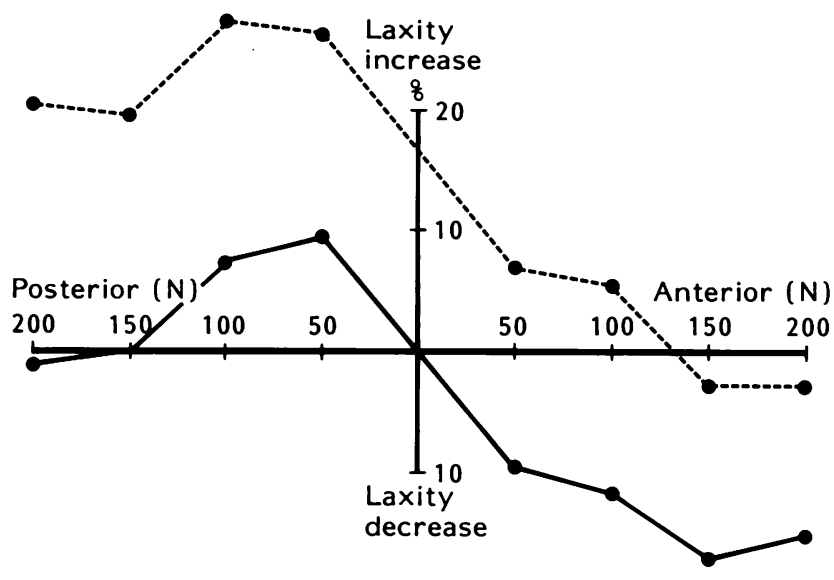

Figure 2. Force (Newton) versus anterior and posterior tibial displacement at 90 degrees of knee flexion: percentage increase or decrease of mean relative to pre-exercise mean values (20 knees)

- - Immediately post-exercise

-30 minutes post-exercise

increase (6.3 per cent). However, a significantly $(P<0.025)$ increased axial tibial rotation post-exercise was observed with walking (15 per cent). Figure 3 illustrates anterior tibial displacement pre- and postexercise during eccentric quadriceps activity. The maximum mean increase (18 per cent) over pre-exercise levels was found at 30 degrees of knee flexion.

\section{Discussion}

Weisman et al. studied effects of competitive exercise on the medial collateral ligament in vivo and found a mean increase in compliance of 22.8 per cent ${ }^{6}$. Stoller et al. determined the effects of exercise on torsional knee laxity and found significant laxity increases of 14 per cent, peaking between 10 to 20 minutes after exercise and returning to normal after 60 minutes ${ }^{5}$. In contrast, post-exercise rotational laxity in our study increased by only 4.9 per cent. However, we applied

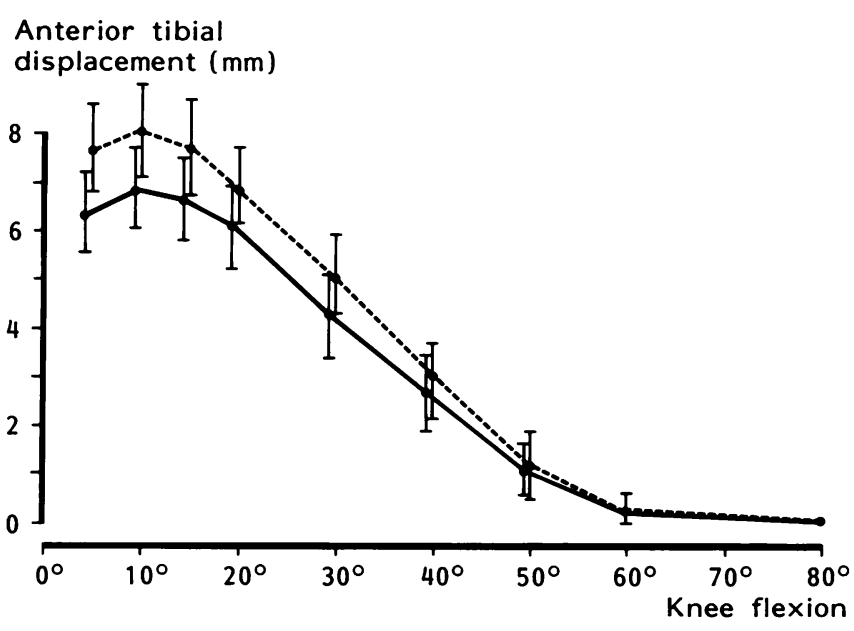

Figure 3. Pre- and post-exercise, anterior tibial displacement during eccentric quadriceps activity with 10 per cent of body weight attached to the foot (anterior tibial displacement with eccentric loaded quadriceps activity (0 to 90 degrees of knee flexion) relative to tibial displacement during unloaded, passive, relaxed knee flexion). Mean values and one standard deviation (20 knees)

- pre-exercise

- - - post-exercise

considerably less torque and the rotational laxity tests were performed within the first five minutes postexercise. We may therefore have missed the peak values.

Using Stryker's knee laxity tester, Steiner et al. tested a group of distance runners and found postexercise, passive, antero-posterior laxity increases between 18 and 20 per cent at 20 degrees of knee flexion ${ }^{4}$. In comparison we found increases between 15 and 16 per cent at 30 degrees of knee flexion. Skinner et al., using a knee arthrometer (KT 1000) to quantify anterior-posterior knee laxity, found less anteriorposterior laxity increase post-exercise (11 per cent $)^{3}$. However, their exercise protocol was somewhat different, with recovery periods interspersed, making direct comparisons difficult.

Based on these studies and the findings of the present study, it seems fairly conclusive that exercise does increase knee joint laxity, both torsional, valgus-varus and anterior-posterior laxity. However, the cause of this laxity increase is unclear. Theoretically, there are at least two possible sources. It could be caused by ligamentous laxity resulting from the exercise, by decreased muscle resting tone from fatigue, or possibly by the combined influence of these factors.

Animal studies have documented that ligaments respond to physiological stresses in the form of training in running machines or long term jumping exercises by increasing strength, and conversely that immobilization causes a reduction in strength ${ }^{7-9}$. The fact that insufficiency of the cruciate or collateral ligaments cause symptoms can be taken as indirect evidence that these ligaments are subjected to significant loading in vivo by many activities of daily living.

More direct evidence has been provided by Henning et al. ${ }^{10}$. In an in vivo study of the load-elongation characteristics of grade II anterior cruciate ligament sprains using strain gauges, they found significant 
elongation of the anterior cruciate ligament with many activities not normally considered strenuous (jogging, walking, quadriceps exercise). Maximum elongation was found with down-hill running.

While these studies indicate that ligaments are subjected to significant stress under physiological conditions, they do not prove that this stress can actually produce significant elongation of the ligamentous restraints of the knee.

Theoretically, capsular structures and ligaments in and around the knee could be expected to demonstrate visco-elastic behaviour, responding with elongation phenomena to repetitive stress, as a primary constituent of these structures is collagenous tissue. Although no definite proof of this theory has been supplied, several studies indicate that the laxity increase observed post-exercise is indeed caused by an increase in ligamentous laxity, rather than the result of decreased muscle resting tone caused by muscular fatigue.

Steiner et al. measured anterior-posterior laxity before and during general anaesthesia and found no difference ${ }^{4}$, indicating that functionally complete muscle relaxation can be obtained during testing of an unanaesthetized individual, and that the influence of muscle tonus can therefore be disregarded. However, only 12 knees were tested in 11 individuals, so this observation may, therefore, not be universally true.

Also suggestive of a ligamentous response, rather than a muscle response, are the findings of Stoller et $a l .5$. They found differences in the rate of return to normal laxity due to physical modalities - application of ice, heat or ultrasound to the knee - not the musculature around the knee. In our study, the post-exercise anterior-posterior laxity increase observed with eccentric quadriceps activity would also be difficult to explain if an increased ligamentous laxity is not assumed.

In an in vitro study using cadaver knees, Markolf $e t$ al. determined the effect of joint load on anterior-posterior laxity at 20 degrees of flexion, and found changes of about one millimetre with a change in joint load of 100 Newton $^{11}$. A decrease in joint load of 100 Newton due to muscle fatigue post-exercise could account for the increased laxity observed in our study.

In part, the results of this study and the study by Skinner et al. indicate a modifying influence of muscle tonus on post-exercise laxity in vivo ${ }^{3}$. Skinner et al. subjected the athletes' right knees to both sprinting and isokinetic exercises, whereas the left knee was subjected to sprinting exercises only with a recovery period, while the contralateral knee was isokinetically exercised. Under these circumstances, they found post-exercise laxity increase in the left knee only. This suggested that increased joint load, due to increased resting muscle tone in the isokinetically exercised muscles, caused the lack of post-exercise laxity increase in the right knees.

In our study, explaining the pattern of anteroposterior post-exercise laxity increases at 30 and 90 degrees of knee flexion and the subsequent laxity decreases after 30 minutes of recovery would be difficult if a modifying influence of muscle tone was not assumed (Figure 1 and Figure 2). More uniform increases throughout the range of applied force, regardless of the direction of the force applied, would be expected, as would a more uniform decrease following the recovery period.

Pre- and post-exercise values for peak force during isometric quadriceps and hamstring exercises were not measured in our study. A possible explanation of the lack of increase in post-exercise antero-posterior laxity during these tests could be a decrease in quadriceps and hamstring force because of fatigue, as indicated by Sherman et al., who found 30 per cent to 35 per cent reduced quadriceps and hamstring strength after a marathon ${ }^{12}$. The unchanged laxity could therefore represent an actual increase, in that it was caused by exertion of less muscular force.

In conclusion, exercise induces transient significant increase in knee joint laxity. The exact mechanism of the increased laxity is unknown. The most probable explanation seems to be a true ligamentous laxity increase, modified by alterations in muscle tone.

\section{References}

1 Abrahams, M. Mechanical behavior of tendon in vitro Med Biol Eng 1967, 5, 433-443

2 Hooley, C.J., and McCrum, N.G. The viscoelastic deformation of tendon J Biomech 1980, 13, 521-528

3 Skinner, H.B., Wyatt, M.P., Stone, M.L et al. Exerciserelated knee joint laxity Am J Sports Med 1986, 14, 30-34

4 Steiner, M.E., Grana, W.A., Chillag, K. et al. The effect of exercise on anterior-posterior knee laxity Am J Sports Med 1986, 14, 24-29

5 Stoller, D.W., Markolf, K.L., Fagen, S.A. et al. The effects of exercise, ice, and ultrasonography on torsional laxity of the knee Clin Orthop 1983, 174, 172-180

6 Weismann, D., Pope, M.H., Johnston, R.J. Cyclic loading in knee ligament injuries Am J Sports Med 1980, 8, 24-30

7 Viidik, A. Elasticity and tensile strength of the anterior cruciate ligament in rabbits as influenced by training Acta Physiol Scand 1968, 74, 372-380

8 Tipton, C.M., Mathes, R.D., Maynard, J.A. et al. The influence of physical activity on ligaments and tendons Med Sci Sports 1975, 7, 165-175

9 Noyes, F.R. Functional properties of knee ligaments and alterations induced by immobilization Clin Orthop $1977,123,210-258$

10 Henning, C.E., Lynch, M.A., Glick, K.R. An in vivo strain gauge study of elongation of the anterior cruciate ligament Am J Sports Med 1985, 13, 22-26

11 Markolf, K.L., Bargar, W.L., Shoemaker, S.C. et al. 1981 The role of joint load in knee stability J Bone Joint Surg 1981, 63A, 570-585

12 Shermann, W.M., Armstrong, L.E., Murray, T.M. et al. Recovery of muscular strength, power, and work capacity following intense endurance exercise: The marathon Med Sci Sports Exerc 1982, 14, 135 